\title{
Análisis de la toxicidad por aluminio en el crecimiento de dos variedades de "frijol", Phaseolus vulgaris $L$.
}

\author{
Analysis of aluminum toxicity in the growth of two varieties of "bean", \\ Phaseolus vulgaris $L$.
}

Recibido: octubre 06 de 2020 | Revisado: agosto 20 de 2020 | Aceptado: diciembre 12 de 2020

\author{
Julio Chico Ruíz ${ }^{1}$ \\ Lisi Cerna Rebaza ${ }^{2}$ \\ Luis GonZales Llontop ${ }^{3}$ \\ Joseph Campos Ruíz ${ }^{4}$ \\ Manuel Rodríguez LacherRes
}

\begin{abstract}
Resumen
Los suelos ácidos constituyen aproximadamente el 35\% de los suelos cultivables y una de las más importantes limitaciones en la agricultura. La restricción en el crecimiento y producción del cultivo con $\mathrm{pH}$ menor a 5.0 es debido, principalmente, a la formación y migración de iones de aluminio $\left(\mathrm{Al}^{3+}\right)$. Con estos antecedentes, se analizó la toxicidad por aluminio en el crecimiento de dos variedades de Phaseolus vulgaris $L$. var. "canario" y var. "panamito". El diseño experimental de bloques completamente al azar tuvo los tratamientos de $50 \mu \mathrm{M}, 25 \mu \mathrm{M}$ y 0 $\mu \mathrm{M}$, con tres repeticiones cada uno. Las plántulas fueron adaptadas a un sistema hidropónico, el cual contenía el medio nutritivo de Hoagland modificado y las diferentes concentraciones de aluminio. Después de tres días se evaluó el peso fresco, peso seco y acumulación del metal en hojas y raíces, la actividad de la peroxidasa, el porcentaje de proteínas y la longitud de las raíces. La variedad "panamito" fue más sensible en relación a la variedad "canario" según las variables evaluadas. La acumulación del aluminio fue mayor en las hojas de "panamito" y en las raíces para la otra variedad. Se concluye que la variedad "canario" es más tolerante que la variedad "panamito".
\end{abstract}

Palabras clave: toxicidad, Phaseolus vulgaris L., aluminio

\begin{abstract}
Acidic soils are approximately $35 \%$ of arable soils and one of the most important limitations in agriculture. The restriction in the growth and production of the culture with a $\mathrm{pH}$ lower than 5.0 is mainly due to the formation and migration of aluminum ions ( $\mathrm{Al} 3$ + ). With this background, the growth toxicity of two varieties of Phaseolus vulgaris L. var. "canario" and var. "panamito" was analyzed. The completely randomized experimental block design had the
\end{abstract}

Autor para correspondencia: jchico@unitru. edu.pe

(C) Los autores. Este artículo es publicado por la Revista Campus de la Facultad de Ingeniería y Arquitectura de la Universidad de San Martín de Porres. Este artículo se distribuye en los términos de la Licencia Creative Commons Atribución No-comercial - Compartir-Igual 4.0 Internacional (https://creativecommons.org/licenses/ CC-BY), que permite el uso no comercial, distribución y reproducción en cualquier medio siempre que la obra original sea debidamente citada. Para uso comercial contactar a: revistacampus@usmp.pe. 
treatments of $50 \mu \mathrm{M}, 25 \mu \mathrm{M}$ and $0 \mu \mathrm{M}$, with three repetitions each. The seedlings were adapted in a hydroponic system, which contained the modified Hoagland nutrient medium and the different concentrations of aluminum. After three days root length, fresh and dry weight, accumulation of the metal in leaves and roots, peroxidase activity, protein percentage were evaluated. The panamito was more sensitive in relation to the canario according to the evaluated variables. Aluminum accumulation was greater in the leaves for the panamito and in the roots for the other variety. It is concluded that the canario is more tolerant than the panamito variety.

Key words: toxicity, Phaseolus vulgaris L., aluminum

\section{Introducción}

La toxicidad de los metales en el suelo no depende de la concentración total, sino de su biodisponibilidad. Entre los factores más relevantes para la disponibilidad podemos mencionar el $\mathrm{pH}$, el potencial de óxido reducción, la capacidad de intercambio catiónico y la materia orgánica. Por regla general, en un suelo ácido, la forma de catión libre del aluminio $\left(\mathrm{Al}^{3+}\right)$ suele ser la forma más tóxica (Snyman et al., 2019; Poschenrieder \& Barceló, 2004).

El aluminio (Al) es el tercer elemento más abundante que representa alrededor del $8 \%$ de la corteza terrestre. En la litosfera, nunca se encuentra como un metal puro; pero, sí en combinación con oxígeno y silicio, formando aluminiosilicatos complejos e insolubles, los cuales son abundantes en rocas ígneas, metamórficas y sedimentarias (Pereira, 2018; Reigosa et al., 2004). En suelos neutros o alcalinos no se alcanzan concentraciones tóxicas para los vegetales; sin embargo, con la reducción del $\mathrm{pH}$ del suelo se incrementa la solubilidad del aluminio hasta llegar a ocupar más de la mitad de los sitios de intercambio iónico del suelo (Zhao \& Shen, 2018; Prieto, 2009; Caiserra \& Aguilar, 2007).

El aluminio es absorbido con gran rapidez por las raíces; debido, a que los complejos de aluminio como $\mathrm{Al}(\mathrm{OH})_{3} \cdot 3 \mathrm{H}_{2} \mathrm{O}$ o $\mathrm{AlCl}_{3}$ son más móviles y atraviesan la membrana plasmática a través de los poros hidrófilos o por los canales de proteína y así alcanzan el interior de la célula (Rao et al., 2016; Caiserra \& Aguilar, 2007), y desde allí comienzan a interrumpir los procesos fisiológicos a nivel celular y molecular, incluyendo captación de agua y nutrientes, transporte intracelular, división y elongación celular, señales celulares y homeostasis redox (Pereira, 2018; Barcelo \& Poschenrieder, 2002), siendo el principal síntoma de toxicidad la inhibición de la elongación de la raíz (Kochian et al., 2015). Otros mecanismos de toxicidad implican a la pared celular (Zhang et al., 2016) y a la membrana plasmática que modifican su estructura, causando problemas en el transporte de iones y un desbalance de los mismos (Bose et al., 2011); también, afecta la constitución del simplasto (calmodulina) (Tokizawa et al., 2015), el apoplasto (matriz de la pectina (Delhaize et al., 2007) y el ADN de las células de la raíz (Sade et al., 2016) o 
a nivel de suelo se une al fósforo $(\mathrm{P})$ y no está disponible para las raíces y se vuelve insoluble (Kochian et al., 2004).

La sintomatología por toxicidad con $\mathrm{Al}$ se divide en dos categorías: (1) respuestas largas que requiere horas para manifestarse, y (2) respuestas cortas que se pueden medir en minutos o en segundos después de exponerse a $\mathrm{Al}$ (Simoes et al., 2012). El primer síntoma perceptible es la reducción del crecimiento radicular en longitud, efecto que en Triticum aestivum empieza una hora después asociado con un incremento del diámetro de los ápices radiculares (Caiserra \& Aguilar, 2007). Zhang et al., (2016) trabajando con $P$. vulgaris investigó la interacción entre el estrés hídrico y la toxicidad por aluminio, encontrando nuevos mecanismos fisiológicos y moleculares que modifican la estructura de la pared celular de la raíz como respuesta al estrés osmótico inducidos por polietilenglicol (PEG) y el aluminio. Eticha et al., (2010) afirma que en la resistencia del frijol al aluminio interviene el citrato.

Phaseolus vulgaris L. "frijol" es un cultivo muy apreciado por sus semillas que son de consumo popular, además es una planta muy sensible a las condiciones medio ambientales adversas, por lo que su cultivo se limita a ciertas áreas que llevan los requisitos de clima y suelos nutritivos que exige la planta (Mallor et al., 2018; Cavalieri et al., 2011; Ramírez, 2014). La mayor parte de su producción a nivel mundial se presenta en los países de bajos recursos, como los pertenecientes a los del continente americano y africano. En el Perú es importante en el aspecto de sostenibilidad económica, social y ambiental. Actualmente, representa un cultivo con posibilidades económicas en la población rural como una fuente importante de ocupación e ingreso, debido al potencial exportador de ciertas variedades; así como, una garantía de seguridad alimentaria vía autoconsumo (Valladolid \& Voyset, 2006; González, 2008; Ministerio de Agricultura, 2010; 2016).

A consecuencia del incremento de concentraciones de metales en los suelos, los aumentos de la biodisponibilidad de los mismos para múltiples cultivos han estado causando daños de fitotoxicidad, provocando un riesgo para la salud de animales y los hombres. Entonces es necesario conocer su eficiencia para acumular y resistir a los metales en sus tejidos (Jacob et al., 2016); además, con sus características de tallo corto (menos de un metro), raíces fibrosas, grandes y gruesas, y de ciclo biológico corto (tres meses aproximadamente) nos permite desarrollar experiencias en el laboratorio, pues la respuesta fisiológica a una situación estresante es rápida.

Por todos estos antecedentes, en la presente experiencia se planteó como objetivo analizar la toxicidad por aluminio en el crecimiento de dos variedades de Phaseolus vulgaris L. variedad "canario" y variedad "panamito" en condiciones de laboratorio, en la cual se consideraron las variables: biomasa fresca y seca de hojas y raíz, longitud de la raíz, contenido de aluminio y proteínas en hojas y raíces, y la actividad de la peroxidasa.

\section{Método}

Las semillas de Phaseolus vulgaris L. variedad "canario" y "panamito" fueron adquiridas en la estación experimental, EEA "Villa Florida" (INIA) con sede en Chiclayo-Perú. La experiencia se 
realizó en condiciones de laboratorio. Primero se puso a germinar 100 semillas (previa desinfección con lejía al 2\%) en placas Petri (aprox. 20 semillas por placa), en cuyo interior había papel toalla humedecido con agua destilada $(20 \mathrm{ml})$. Las plántulas con cinco días de edad y raíces con longitudes de $5 \mathrm{~cm}$ fueron seleccionadas para la experiencia. Luego, se adaptaron al sistema hidropónico, el cual consistió en recipientes de plástico $(40 \times 15 \times 15 \mathrm{~cm})$ con cuatro litros de agua destilada más el medio nutritivo (medio de Hoagland modificado, pH 6.5. Las plántulas se suspendieron en planchas de tecnopor que tuvieron 10 agujeros de $2 \mathrm{~cm}$ de diámetro (un agujero para cada plántula); todo el sistema estuvo aireado con bombas de acuario.

Se utilizó el diseńo experimental de bloques completamente al azar con tres tratamientos y tres repeticiones (cada unidad experimental con 10 plántulas, con un total de 60 plántulas por bloque). Como fuente de aluminio se utilizó $\mathrm{AlCl}_{3}$ en las concentraciones de $[0 \mu \mathrm{M}]$, $[25 \mu \mathrm{M}]$ y $[50 \mu \mathrm{M}]$. Después de siete días de estar en el sistema hidropónico, se inició la experiencia durante tres días. Se hicieron las siguientes evaluaciones:

\section{a. Biomasa fresca y seca de hojas y raíz} En una balanza analítica, se determinó el peso fresco de hojas y raíz de 10 plantas, después se llevó a la estufa por 24 horas a $65^{\circ} \mathrm{C}$ y se determinó el peso seco de ambos órganos vegetales.

\section{b. Longitud de raíz}

Se midió la raíz más larga utilizando una regla milimetrada para cada uno de los tratamientos. Los datos fueron convertidos mediante la siguiente fórmula que permite obtener la elongación relativa de la raíz:
$E R R=\frac{\text { Longitud de la raíz con aluminio }}{\text { Longitud de la raíz sin aluminio }} X \quad 100$

\section{c. Contenido de aluminio en hojas y raíces}

Se analizaron las muestras secas de hojas y raíces expuestas a diferentes concentraciones de $\mathrm{AlCl}_{3}$ mediante el método de espectrofotometría de absorción atómica. Los resultados se expresaron en unidades de $\mathrm{mg} \mathrm{Al} / \mathrm{g}$.

\section{d. Actividad de la peroxidasa * Obtención del extracto crudo de peroxidasa}

Las raíces de "frijol" se lavaron con agua destilada de tres a cuatro veces para eliminar impurezas y refrigeradas por 24 horas a una temperatura de $13^{\circ} \mathrm{C}$; luego, se trituró con un mortero, de los cuales se obtuvo un extracto crudo, el cual fue filtrado tres veces en papel de filtro para luego llevarlo y guardarlo a $8^{\circ} \mathrm{C}$ (Arellano et al., 2013).

\section{Medición de la actividad de la peroxidasa}

El extracto crudo se acondicionó con buffer fosfato $0.1 \mathrm{M}$ de $\mathrm{pH}$ 7.0, para luego realizar las mediciones de absorbancia en un espectrofotómetro Termocientific evolution $300 \mathrm{UV}-\mathrm{Vis}$ a una longitud de onda de $470 \mathrm{~nm}$; los sustratos utilizados fueron guayacol $16 \mathrm{mM}$ y peróxido de hidrógeno $\left(\mathrm{H}_{2} \mathrm{O}_{2}\right) 8 \mathrm{mM}$. Se agregaron $25 \mu \mathrm{L}$ de extracto crudo a una cubeta de cuarzo, el cual contenía los sustratos $0.5 \mathrm{ml}$ de guayacol $16 \mathrm{mM}$ y $0.5 \mathrm{ml}$ de peróxido de hidrógeno $8 \mathrm{mM}$, ambos diluidos en buffer fosfato $0.1 \mathrm{M}$ de $\mathrm{pH}$ 6.8. (Civello et al., 1995). 


\section{e. Determinación de proteínas}

A partir de la muestra seca se procedió a la determinación de proteínas a las 0, 24, 48 y 72 horas utilizando la técnica de Kjeldahl (Gerhardt, 2015) Primero, se determinó el porcentaje de nitrógeno presente en la muestra y para encontrar el porcentaje de proteínas se aplicó la siguiente fórmula:

$\%$ proteínas $=\% \mathrm{~N} \times 6.25$
Se utilizó el programa IBM Stadistical Package for the Social Science (SPSS) Statistics 28 para obtener el promedio y desviación estándar y ANOVA de los datos obtenidos, considerando el valor de $\mathrm{p}<0.05$ como significativo para establecer si existe diferencias significativas entre los tratamientos.

\section{Resultados}

Tabla 1

Longitud de raices en dos variedades de P. vulgaris al ser expuestas a diferentes concentraciones de aluminio a las 72 horas. Los resultados son promedios de tres repeticiones y los tratamientos corresponden al aluminio en $\mu M$.

\begin{tabular}{lcccccc}
\hline Tratamiento & \multicolumn{2}{c}{ T1 } & \multicolumn{2}{c}{ T2 } & \multicolumn{2}{c}{ T3 } \\
Variedad & $\mathbf{0}$ & ERR & $\mathbf{2 5}$ & ERR & 50 & ERR \\
\hline Canario & $1.26 \pm 0.20$ & 100 & $0.26 \pm 0.05^{\mathrm{a}}$ & 20.63 & $0.17 \pm 0.05^{\mathrm{a}}$ & 13.49 \\
Panamito & $0.97 \pm 0.27$ & 100 & $0.12 \pm 0.01^{\mathrm{b}}$ & 12.37 & $0.11 \pm 0.06^{\mathrm{b}}$ & 11.34 \\
\hline
\end{tabular}

Nota. Letras diferentes indican diferencias significativas con ANOVA, y $\mathrm{p}<0.05$

ERR: elongación relativa de la raíz

Con respecto a la longitud promedio de raíz en las dos variedades de $P$. vulgaris $L$. al ser expuestas a diferentes concentraciones de aluminio (Tabla 1) se observó que la variedad "canario" logró un mayor crecimiento con $1.26 \mathrm{~cm}$ en T1 (control), y fue disminuyendo su longitud hasta el tratamiento $3(50 \mu \mathrm{M})$ con $0.17 \mathrm{~cm}$ (13.49 ERR). Asimismo, la variedad "panamito" logró un mayor crecimiento con $0.97 \mathrm{~cm}$ en T1 (control), y una disminución en el crecimiento de la longitud hasta T3 con $0.11 \mathrm{~cm}(11.34$ ERR). Estadísticamente, los resultados fueron significativos en la longitud de la raíz, quiere decir que hubo respuestas diferentes en el crecimiento de cada uno de ellos con respecto al tratamiento aplicado.

Las raíces, expuestas a $50 \mu \mathrm{M}$ de $\mathrm{Al}$, al ser coloreadas con azul de metileno (1\%) observamos que las células del borde están destruidas (Fig. 3), la misma situación para la epidermis y la corteza (Fig. 2), en la Fig. 1 se observa la raíz de variedad "canario" con el tejido ligeramente afectado. El daño fue mucho mayor cuando se prolongó el tiempo de exposición (de 24 a 72 horas) y si se incrementaba la concentración de aluminio, las raíces se volvían flácidas y se rompían con facilidad. 
Julio Chico Ruíz - Lici Cerna Rebaza - Luis gonzales Llontop - Joseph Campos Ruíz - Manuel RODRÍGUEZ LACHERRE

Tabla 2

Peso fresco (g.) de las hojas y raices de las variedades canario y panamito al ser expuestas a diferentes concentraciones de aluminio $(\mu M)$ a las 72 horas. Los resultados es el promedio de tres repeticiones.

\begin{tabular}{lllll}
\hline \begin{tabular}{l} 
Variedad \\
\multicolumn{1}{c}{ Tratamiento }
\end{tabular} & Órgano & \multicolumn{1}{c}{ T1 } & \multicolumn{1}{c}{ T2 } & T3 \\
& & 0 & 25 & 50 \\
\hline Canario & \multirow{2}{*}{ Hojas } & $17.83 \pm 4.11$ & $17.3 \pm 5.98(0.53)$ & $14.66 \pm 3.98(3.17)$ \\
Panamito & & $4.68 \pm 0.18$ & $4.62 \pm 0.33(0.06)$ & $4.22 \pm 0.01(0.46)$ \\
Canario & \multirow{2}{*}{ Raíz } & $15.13 \pm 0.37$ & $14.7 \pm 2.75(0.43)$ & $14.43 \pm 5.62(0.7)$ \\
Panamito & & $4.13 \pm 0.67$ & $3.13 \pm 0.46(1)$ & $3.10 \pm 0.99(1.03)$ \\
\hline
\end{tabular}

Los valores en paréntesis son la diferencia con los valores del testigo.

Tabla 3

Peso seco (g.) de las hojas y raices de las variedad canario y panamito al ser expuestas a diferentes concentraciones de aluminio $(\mu \mathrm{M})$ a las 72 horas. Los resultados son promedios de tres repeticiones.

\begin{tabular}{lcccc}
\hline Variedad & Órgano & T1 & T2 & T3 \\
\multicolumn{1}{c}{ Tratamiento } & & 0 & 25 & 50 \\
\hline Canario & \multirow{2}{*}{ Hojas } & $6.56 \pm 0.09$ & $6.33 \pm 044(0.23)$ & $6.2 \pm 0.43(0.36)$ \\
Panamito & & $2.76 \pm 0.09$ & $2.6 \pm 0.08(0.16)$ & $2.53 \pm 0.24(0.23)$ \\
Canario & \multirow{2}{*}{ Raíz } & $5.8 \pm 0.08$ & $5.46 \pm 0.32(0.34)$ & $5.33 \pm 0.24(0.47)$ \\
Panamito & & $2.5 \pm 0.08$ & $2.23 \pm 0.04(0.27)$ & $2.16 \pm .0 .12(0.34)$ \\
\hline
\end{tabular}

Los valores en paréntesis son la diferencia con los valores del testigo.

El peso fresco de las hojas de las dos variedades de P. vulgaris L. al ser expuestas al aluminio perdieron peso según se iba incrementando la concentración del metal. La diferencia en el peso es mayor en la hoja que en la raíz para la variedad "canario". En cambio, con la variedad "panamito" es la raíz la que pierde más peso en T3 (Tabla 2). Con respecto al peso seco de las hojas y raíces, se observó que ambas variedades iban perdiendo peso según se incrementaba la concentración del metal (Tabla 3). Se perdió más peso seco en las raíces de la variedad "canario"; al igual que para la variedad "panamito".

Tabla 4

Acumulación de aluminio ( $m g$ Allg de vegetal) en hojas y raíces de las variedades "canario" y "panamito" a las 72 horas de ser expuestas al metal.

\begin{tabular}{lcccc}
\hline Variedad & Órgano & T1 & T2 & T3 \\
\multicolumn{1}{c}{ Tratamiento } & & 0 & 25 & 50 \\
\hline Canario & \multirow{2}{*}{ Hojas } & $0.009 \pm 0.00$ & $0.042 \pm 0.00^{\mathrm{a}}$ & $0.072 \pm 0.00^{\mathrm{a}}$ \\
Panamito & & $0.032 \pm 0.00$ & $0.161 \pm 0.00^{\mathrm{b}}$ & $1.717 \pm 0.00^{\mathrm{b}}$ \\
Canario & \multirow{2}{*}{ Raíz } & $0.013 \pm 0.00$ & $0.074 \pm 0.00^{\mathrm{c}}$ & $0.130 \pm 0.00^{\mathrm{c}}$ \\
Panamito & & $0.036 \pm 0.00$ & $0.563 \pm 0.00^{\mathrm{d}}$ & $0.218 \pm 0.00^{\mathrm{d}}$ \\
\hline
\end{tabular}

Letras diferentes indican diferencias significativas con ANOVA, y $\quad \mathrm{p}<0.05$

En ambas variedades hubo incremento en el contenido del metal directamente proporcional a la concentración del metal de cada tratamiento (Tabla 4). En la variedad "canario" se acumuló más aluminio en las raíces $(0.130 \mathrm{mg} \mathrm{Al} / \mathrm{g})$. 
En cambio, para la variedad "panamito" el aluminio se acumuló más en las hojas $(1.717 \mathrm{mg} \mathrm{Al} / \mathrm{g})$. También se observó que hay mayor acumulación de aluminio en la variedad "panamito" en las hojas o en las raíces. Estadísticamente, los resultados fueron significativos para la acumulación de aluminio en los órganos vegetales.

Tabla 5

Unidades de la enzima (UE) peroxidasa en raices de la variedad "canario" y variedad "panamito" expuestas a diferentes concentraciones de aluminio ( $\mu M)$

\begin{tabular}{llll}
\hline \multicolumn{1}{c}{ Variedad } & \multicolumn{1}{c}{ T1 } & T2 & T3 \\
\multicolumn{1}{c}{ Tratamientos* } & 0 & 25 & 50 \\
\hline Canario & $270.3 \pm 0.05$ & $16.15 \pm 0.02$ & $15.3 \pm 0.04$ \\
Panamito & $237.15 \pm 0.03$ & $19.9 \pm 0.05$ & $9.35 \pm 0.04$ \\
\hline
\end{tabular}

En las dos variedades se observó una marcada disminución conforme aumenta la concentración en los tratamientos

(T2 y T3), siendo T3 de la variedad "panamito" que presentó el valor más bajo (9.35 UE) (Tabla 5).

Tabla 6

Contenidos de proteinas en raices de las variedades "canario" y "panamito" cuando fueron expuestos a $25 \mu M$ de aluminio durante tres dias.

\begin{tabular}{ccccccccc}
\hline $\begin{array}{c}\text { Tratamiento } \\
(\mathrm{Al}, \mu \mathrm{M})\end{array}$ & \multicolumn{3}{c}{0} & \multicolumn{5}{c}{25} \\
\hline $\begin{array}{c}\text { Horas } \\
\text { Variedad }\end{array}$ & 0 & 24 & 48 & 72 & 0 & 24 & 48 & 72 \\
Canario & 22.5 & 22.65 & 23.05 & 24.15 & 21.9 & 26.8 & 28.1 & 35.0 \\
Panamito & 18.25 & 18.95 & 19.25 & 21.4 & 24.0 & 24.3 & 25.0 & 25.6 \\
\hline
\end{tabular}

Para la variedad "canario" el porcentaje de proteínas aumentó a $35 \%$ a las 72 horas, casi el doble; en cambio, a las 72

horas para la variedad "panamito" llegó a un porcentaje de 25.6 aumentando aproximadamente $1 \%$. (Tabla 6 ).

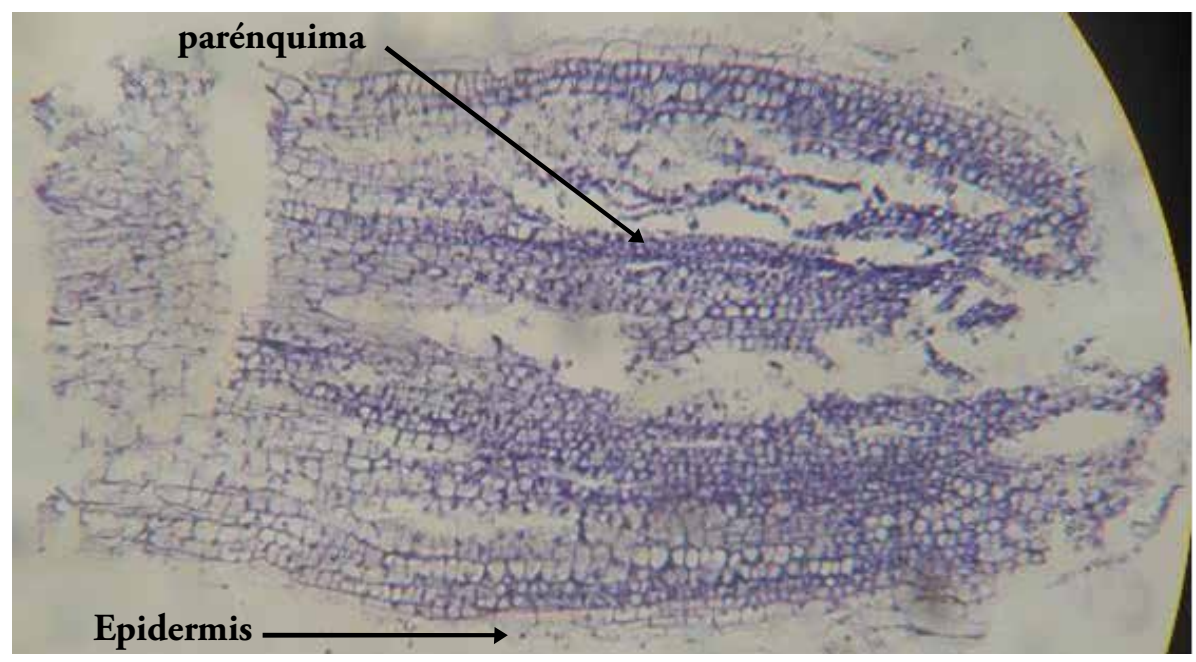

Figura 1. Tejido de ápice de raíz de variedad "canario" a las 24 horas de estar expuesto a cloruro de aluminio $50 \mu \mathrm{M}$. Se observa las células de la epidermis y el tejido parenquimático y conductor ordenado y de tamaño normal. Corte longitudinal, coloración azul de metileno 1\%. 400x 


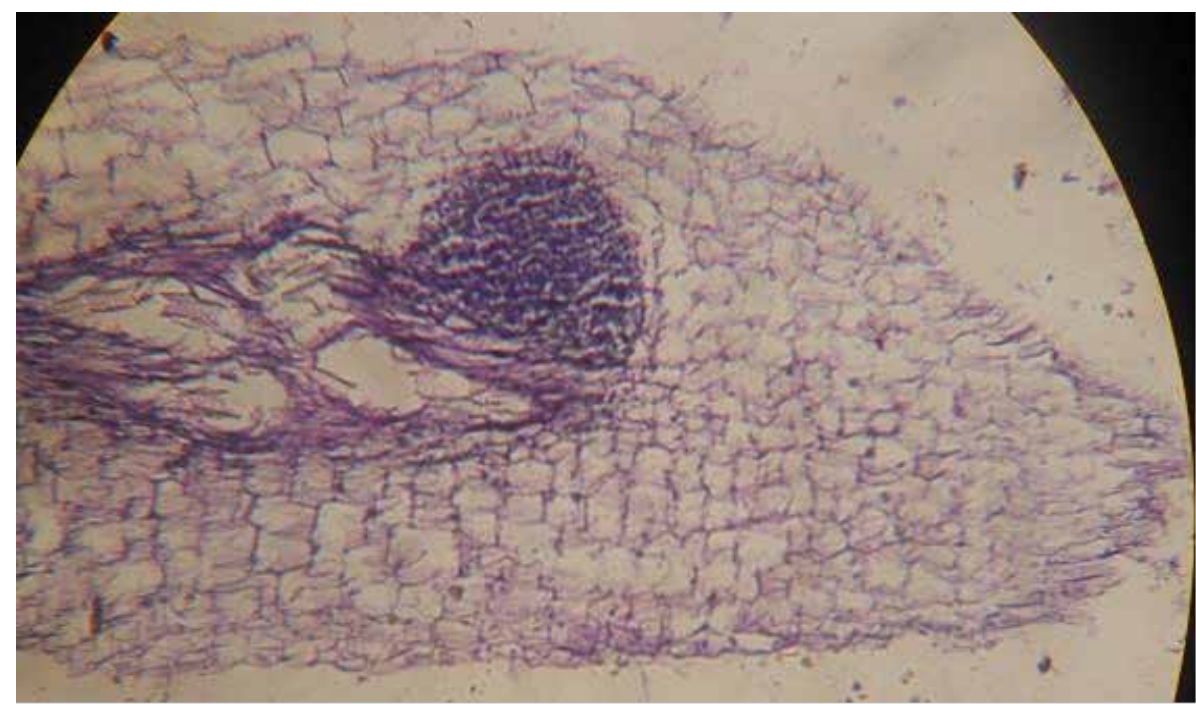

Figura 2. Tejido de ápice de raíz variedad "panamito" a las 48 horas de estar expuesto a aluminio $50 \mu \mathrm{M}$. Se observa tejido desorganizado con células grandes, no existe tejido epidérmico. Corte longitudinal, azul de metileno 1\%. 400x

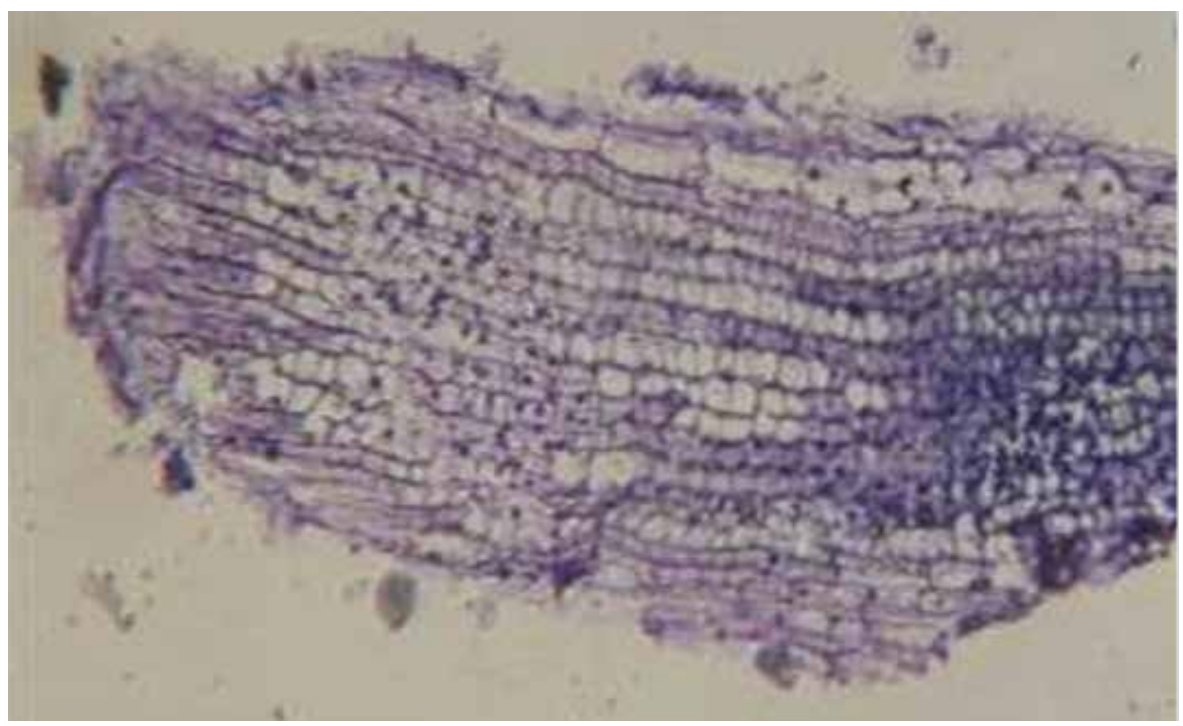

Figura 3. Tejido de ápice de raíz variedad "panamito" a las 24 horas de estar expuesta a aluminio $50 \mu \mathrm{M}$. Raíces ha perdido parte de la epidermis. Corte longitudinal, coloración azul de metileno 1\%. 400x

\section{Discusión}

Los resultados muestran la reducción del crecimiento en longitud de la raíz en $P$. vulgaris como el síntoma inmediato al añadir aluminio (Tabla 1). Ello correspondeala descripción de un síntoma visual no específico, que en nuestro caso se manifestó desde el primer intervalo de exposición al aluminio (T2), y si a ello añadimos que no existió absorción de agua y nutrientes, el peso seco de las raíces fue disminuyendo (Tabla 3). Esto concuerda con trabajos realizados por Chico-Ruiz et al. (2016) quién encontró una inhibición del crecimiento de la raíz en la variedad "caballero" a 100uM de aluminio, o en $T$. aestivum donde se observó un incremento del diámetro y una disminución en la longitud de los ápices radiculares; de manera que, las raíces afectadas por $\mathrm{Al}^{3+}$ muestran un 
volumen muy limitado del suelo y así se reduce su capacidad para tomar los nutrientes y el agua (Casierra \& Aguilar, 2007; Casierra \& Cárdenas, 2007).

Cabe señalar, que la toxicidad por aluminio normalmente disminuye la concentración de ciertos elementos en las plantas. La disminución de la absorción catiónica puede estar correlacionada con la inhibición del crecimiento radicular ya que sobre todo cationes como el $\mathrm{K}+$ cuya acumulación contribuye a la expansión celular ven afectada su absorción en presencia de Al (Kochian et al., 2015). También observamos que los tejidos a nivel de raíz, se desorganizan y las células epidérmicas aumentan de volumen, debido a que es la primera capa de tejido que se expone al aluminio y responde a varios factores de estrés en defensa de la planta; como lo sostiene Jaskowiak et al. (2019). Este autor también afirma que el ápice de la raíz se altera debido a las modificaciones de los tejidos meristemáticos ya que el aluminio actúa directamente sobre la programación de la muerte celular.

La variedad "canario" perdió agua por las hojas y la variedad "panamito" por las raíces, en relación a su peso fresco, según se observa en la Tabla 2; debido, a que las membranas pierden sus propiedades pues a los primeros minutos de exposición de las raíces al aluminio, ésta ya es afectada y el mecanismo de resistencia de la planta comienza a desarrollarse; como lo sostiene Ryan et al., (1993). Numerosos estudios indican que la membrana plasmática es la primera afectada: el $\mathrm{Al}$ se une a los fosfolípidos de la membrana plasmática, altera la permeabilidad y potencial electroquímico de la membrana, interactúa con la cadena de cationes y transportadores de membrana e inhibe la actividad de la H-ATPasa de la membrana plasmática. (Huynh et al., 2012), con una consiguiente disminución de la actividad mitótica; como lo afirma Jaskowiak et al. (2018).

Cuando se analizó en que órgano de la planta se acumula el aluminio, encontramos que en la variedad. "canario" se acumula en las raíces y en la variedad "panamito" en las hojas (Tabla 4); esto se debió, a que esta variedad tiene una alta capacidad de translocación y transpiración por su sensibilidad al aluminio. Según Famoso et al., (2011) el aluminio se acumula en las raíces y simplasto de las hojas como Al-citrato no tóxico o $\mathrm{Al}$-oxalato, y por el xilema se traslada como citrato más que oxalato según Ma \& Hiradate, (2000). Se indica además, que el aluminio intercambia de oxalato a citrato cuando es cargado en el xilema y cambia a oxalato cuando se traslada a las hojas o se restringe la acumulación de otros elementos minerales en las hojas, lo cual sugiere que plantas que acumulan aluminio han desarrollado mecanismos eficientes para usar bajas concentraciones; como lo afirma Kochian et al., (2015).

La diferencia encontrada en la acumulación de ambas variedades es notoria, debido a que en la variedad "canario", en ambos tratamientos expuestos con aluminio, las plántulas siguen acumulando el metal, en contraste con la variedad "panamito" donde se observó una disminución en la acumulación del metal en el T3 (50ppm); debido, a la muerte de varias plántulas o a la falta de transporte de nutrientes de las raíces a las hojas como consecuencia del menor grado de resistencia a este metal. 
La enzima peroxidasa fue disminuyendo, en UE, conforme aumenta las concentraciones en los tratamientos. Esto se debió a que el aluminio actuó como un inhibidor en la actividad enzimática (Tabla 5), pues existe una unión del aluminio a otros constituyentes de la membrana como enzimas, extensinas o xiloglucanos, los cuales probablemente no afecten a las propiedades físicas de la pared, pero sí a su funcionalidad; es decir, provocar una inhibición entre el sustrato y la enzima, evitando su unión; como lo afirma Yamunarani et al., (2004) o también la presencia del aluminio induce la acumulación local de $\mathrm{H}_{2} \mathrm{O}_{2}$ lo cual modula el estado redox de las peroxidasas, malato deshidrogenasas, thiol asociado a proteínas redox, quitinasas y PR-1; como lo sostiene Zhou et al., (2011). En relación a la presencia de proteínas, observamos altos porcentajes en raíces de las variedades de frejol estudiadas (Tabla 6). Al respecto, Palacios-Castillo et al. (2016) encontró que a $25 \mathrm{uM}$ de aluminio el porcentaje de proteínas fue elevado y se relacionaba con su resistencia. De igual opinión, Zhou et al (2011) reportó la expresión de unas 54 proteínas en apoplastos de raíces de arroz y muchas de ellas implicadas en el metabolismo del carbohidrato.

\section{Conclusiones}

Las variedades "canario" y "panamito" de $P$ vulgaris $L$. estudiadas, alteran su crecimiento a $50 \mu \mathrm{M}$ de aluminio. La variedad "canario" se presenta más tolerante a la toxicidad por aluminio que la variedad "panamito". El aluminio afecta la longitud de la raíz, disminuye el peso fresco y seco de las hojas y raíces de las dos variedades de $P$. vulgaris. $L$. Las raíces de la variedad "canario" manifestaron 35\% de proteínas más que el control, a las 72 horas de estar expuestas al aluminio. El aluminio inhibe la actividad enzimática de la peroxidasa de las variedades de P. vulgaris. L.: “canario" (94.33\%) y "panamito" (96.54\%) a 50 $\mu \mathrm{M}$

\section{Referencias}

Arellano, B., Ilich, Z., Salazar, C., Rodríguez, H. (2013). Actividad enzimática y patrones electroforéticos de peroxidasa de raíces de Brassica napus y Raphanus sativus y frutos de Sechium edule y Cucumis sativus. Rebiol 33 (1): $1-10$.

Barceló, J., Poschenrieder, C. (2002). Fast root growth responses, root exudates and internal detoxification as clues to the mechanisms of aluminum toxicity and resistance: a review. Environ Exp Bot 48:75-92
Bose, J., Babourina, O., Rengel, Z Z. (2011). Role of magnesium in alleviation of aluminium toxicity in plants. J. Exp. Bot. 62, 2251-2264.

Casierra, F., Aguilar, O. (2007). Estrés por aluminio en plantas: reacciones en el suelo, síntomas en vegetales y posibilidades de corrección. Revista Colombiana de Ciencias Hortícolas 1 (2): 246-257.

Casierra, F., Cárdenas, J. (2007). Influencia del aluminio sobre el crecimiento de la raíz en coliflor 
(Brassica oleracea L., var. botrytis, Hib. 'Nevada F1'). Revista UDCA Actualidad \& Divulgación Cientifica 10(1), 149-157.

Cavalieri A., Merchant A., van Volkenburgh E. (2011). Why not beans? Functional Plant Biology, 38, iii-vi Foreword

Civello, P., Martínez, G., Chaves, A., Añon, M. (1995). Peroxidase from strawberry fruit (Fragaria ananassa Duch): Partial purification and determination of some properties. J Agric Food Chem. Vol 43 (10): 2596-2601

Chico-Ruíz, J., Cerna-Rebaza, L., Hernández-Villalobos, K., SilvaPereda, L., Vasquéz-Cunya, F. (2016). Efecto del aluminio y el $\mathrm{pH}$ en el crecimiento de raíces de Phaseolus vulgaris var. caballero en condiciones de laboratorio Rebiol 36(2): $4-15$.

Delhaize, E., Gruber, B., Ryan, P. (2007). The roles of organic anion permeases in aluminium resistance and mineral nutrition. FEBS Lett. 581, 2255-2262.

Eticha, D., Zahn, M., Bremer, M., Yang, M., Rangel, A., Rao, I.,Horst, W. (2010). Transcriptomic analysis reveals differential gene expression in response to aluminium in common bean (Phaseolus vulgaris) genotypes. Annals of Botany 105: 1119-1128

Famoso, A., Zhao, K., Clark, R., Tung, C., Wright, M., et al. (2011). Genetic architecture of aluminum tolerance in rice (Oryza sativa) determined through genome-wide association analysis and QTL mapping. PLOS Genet. 7: e1002221

Gerhardt C. (2015). Análisis de Nitrógeno. El método de Johan Kjeldahl. Compendio. Analytical Systems. GMBH \& CO. KG KG. Alemania ps://www.gerhardt.de/ fileadmin/Redaktion/downloads/ Stickstoffanalyse_Die_Methode_ von_Johan_Kjeldahl_gekuerzt_f_ Homepage-spa-ES.pdf

González, L. (2008). Evaluación de la diversidad genética en una colección de germoplasma de frijol común (Phaseolus vulgaris L.) de Ruanda (África). [Tesis]. Pontificia Universidad Javeriana. BogotáColombia.

Huynh, V., Repellina, A., Zuily-Fodila, Y., Pham-Thia, A. (2012). Aluminum stress response in rice: effects on membrane lipid composition and expression of lipid biosynthesis genes. Physiologia Plantarum 146: 272-284.

Jacob, C., Carrasco, B., Schwember, A. (2016). Advances in breeding and biotechnology of legume crops. Plant Cell Tiss Organ Cult 127:561-584

Jaskowiak, J., Kwasniewska, J., MilewskaHendel, A., Kurczynska, E., Szurman-Zubrzycka, M., Szareiko, I. (2019). Aluminum Alters the Histology and Pectin Cell Wall Composition of Barley Roots. Int. J. Mol. Sci. 20, 3039 
Jaskowiak, J.; Tkaczyk, O.; Slota, M.; Kwasniewska, J.; Szarejko, I. (2018). Analysis of aluminum toxicity in Hordeum vulgare roots with an emphasis on DNA integrity and cell cycle. PLoS ONE 13 (2):e0193156.

Kochian, L., Piñeros, M., Liu, J., Magalhaes, J. (2015). Plant adaptation to acid soils: the molecular basis for crop aluminum resistance. Annu. Rev. Plant Biol. 66, 571-598.

Kochian, L., Hoekenga, O., Pineros, M. (2004). How do crop plants tolerate acid soils?- mechanisms of aluminium tolerance and phosphorous efficiency. Annu. Rev. Plant Biol. 55, 459-493.

Ma, J., Hiradate, S. (2000). Form of aluminium for uptake and translocation in buckwheat (Fagopyrum esculentum Moench). Planta 211:355-60

Mallor, C., Barberán, M., Albar, J. (2018). Recovery of a Common Bean Landrace (Phaseolus vulgaris L.) for Commercial Purposes. Front. Plant Sci. 9:1440.

Ministerio de Agricultura y Riego. (2016). Leguminosas de grano. Cultivares y Clases Comerciales del Perú. GALU GRAF S.A.C. LimaPerú

Ministerio de Agricultura-DGPA. (2010). Plan estratégico de la cadena productiva de menestras [Boletín online]. Perú. [Consultado 20 mayo 2018]. Disponible en: http://www.planeamientoygestion. com.pe/consultoria/images/ stories/herramientas/documentos/ menestras.pdf. 2010.

Palacios-Castillo, J., Cerna-Rebaza, L., Chico-Ruíz, J., Collantes-Silva, L.(2011). Efecto del aluminio sobre el crecimiento de Phaseolus vulgaris variedades canario, panamito y red kidney. Rebiol 31(2)

Pereira J.(2018). Initial root length in wheat is highly correlated with acid soil tolerance in the field. Sci. Agric. 75 (1) :79-83

Poschenrieder, C., Barceló, J. (2004). Estrés por metales pesados. En "La Ecofisiología vegetal: Una ciencia de síntesis". Reigosa, M; Pedral, N. y Sánchez, A. (eds.). Thomson Editores Spain Paraninfo, S.A. Madrid. 413-442 p.

Prieto, J. (2009). Contaminacion y fitotoxicidad en plantas por metales pesados provenientes de suelos y agua. Tropical and Subtropical Agrosystems 10: 29- 44

Rao, I., Miles, J., Beebe, S., Horst, W. (2016) Root adaptations to soils with low fertility and aluminium toxicity. Ann Bot 118:593-605

Reigosa, M., Pedro, N.; Sánchez, A. (2004). La Ecofisiología Vegetal. Una ciencia de síntesis. Thomson Editores Spain Paraninfo, S.A. Madrid. España

Ramírez-Torres, L. (2014). Producción de seis variedades de frijol (Phaseolus vulgaris L.) en condiciones 
agroclimáticas del sector Charcape en el valle Jequetepeque. Pueblo cont. 25(2): 123-133

Ryan, P., DiTomaso, J., Kochian, L. (1993). Aluminium toxicity in roots: an investigation of spatial sensitivity and the role of the root cap. J Exp Bot 44: 437-446

Sade, H., Meriga, B., Surapu, V., Gadi, J., Sunita, M. S. L., Suravajhala, P. (2016). Toxicity and tolerance of aluminium in plants: tailoring plants to suit to acid soils. Biometals 29, 187-210.

Simoes, C., Melo, J., Magalhaes, J., Guimaraes, C. (2012). Genetic and molecular mechanisms of aluminum tolerance in plants. Genet. Mol. Res. 11, 1949-1957.

Snyman, S., Naidoo, M., Watt, M., Rutherford, R. (2019). An in vitro screening system to assess aluminum toxicity in sugarcane (Saccharum spp.) cultivars In Vitro Cellular \& Developmental Biology Plant 55: 403-408

Tokizawa, M., Kobayashi, Y., Saito, T., Kobayashi, M., Luchi, S., Nomoto, M. (2015). Sensitive to proton rhizotoxicity 1 , calmodulin binding transcription activator2, and other transcription factors are involved in aluminum-activated malate transporter1. Plant Physiology 167:991-1003
Valladolid, A., Voysest, O. (2006). Clases comerciales de leguminosas de grano. Catálogo para orientar la comercialización en los mercados nacionales e internacionales, PROMENESTRAS TEXChiclayo-Perú. 250 pp.

Yamunarani, K., Jaganathan, R., Bhaskaran, R., Govindaraju, P., Velazhahan, R. (2004). Induction of early blight resistance in tomato by Quercus infectoria gall extract in association with accumulation of phenolics and defenserelated enzymes. Acta Physiol. Plant. 2004. 26 (3): 281-290.

Zhang, M., Ma, Y., Horst, W., Yang, Z. (2016). Spatial-temporal analysis of polyethylene glycol-reduced aluminium accumulation and xyloglucan endotransglucosylase action in root tips of common bean (Phaseolus vulgaris). Ann. Bot. 118, 1-9.

Zhao, X., Shen, R. (2018) Aluminiumnitrogen interactions in the soilplant system. Front Plant Sci 9:807

Zhou, L., Bokhari, S., Dong, C., Liu, J. (2011). Comparative proteomics analysis of the root apoplasts of rice seedlings in response to hydrogen peroxide. PLoS ONE 6:e16723 
